\section{The enlarged extraocular muscle: to relax, reflect or refer?}

\begin{abstract}
Purpose Extraocular muscle enlargement (EOME) is most commonly associated with thyroid eye disease, but there are other causes. We report our outcomes of investigating and managing non-thyroidrelated EOME (NTR-EOME).

Methods Retrospective consecutive case series. Sixteen patients identified by clinical features and orbital imaging. Patient demographics, radiological features, and adjuvant tests including biopsy and final diagnosis were recorded.
\end{abstract}

Results Mean age at presentation 59.3 years (range 24-89 years). Mean follow-up 3.2 years (range 3 months to 5.5 years). Superior rectus (SR) was most commonly involved muscle (8/16 cases) followed by lateral rectus (4/16). Of the 16 cases, 14 were associated with underlying systemic neoplasia (5 lymphoma, 5 metastatic carcinoma, and 4 presumed paraneoplastic syndrome). All SR enlargement was associated with underlying neoplasia. All patients underwent orbital imaging followed by systemic imaging based on clinical index of suspicion (14/16 patients (13 full body CT (FBCT), 1 mammography)). Positive systemic radiological findings were detected in 12/14 cases. Of the remaining 2 patients, 1 underwent full body positron emission tomography-computed tomography (FBPETCT), which detected thyroid carcinoma, and the second patient underwent FBCT for staging following orbital biopsy showing lymphoma. Four patients $(25 \%)$ died within 3 years of follow-up due to disseminated systemic malignancy.

Conclusions All cases of NTR-EOME should be viewed with a high level of clinical suspicion for systemic neoplasia, especially when the SR is involved. FBCT can help to identify a primary systemic cause. FBPET-CT is best reserved for cases negative on FBCT or for staging and monitoring systemic disease. NTR-EOME can be associated with
F Shafi, P Mathewson, P Mehta and HS Ahluwalia

significant mortality (25\%), hence warrants prompt and thorough systemic investigation. Eye (2017) 31, 537-544; doi:10.1038/eye.2016.248; published online 2 December 2016

\section{Introduction}

Extraocular muscle enlargement (EOME) is most commonly associated with thyroid eye disease, however it can also be the resultant manifestation of several other non-thyroidrelated conditions including inflammatory, vascular, neoplastic, infectious, neuromuscular, and metabolic disorders. ${ }^{1}$ We have used the term 'Non-Thyroid-Related-Extraocular Muscle Enlargement' (NTR-EOME) to describe these causes.

The exact incidence of NTR-EOME has been difficult to quantify accurately because of the under-reporting of many cases and disorders, especially those conditions that result in EOME as part of a more diffuse orbital process such as non-specific orbital inflammation and infiltrative tumours. In the largest published review of EOME cases, Lacey et al ${ }^{1}$ described 1849 cases, of which 1750 (95\%) were due to thyroid orbitopathy and only 99 cases (5\%) were associated with other muscle diseases. The commonest causes of NTR-EOME in this series were inflammatory $(45 / 99,45 \%)$, vascular (24/99, 24\%), and neoplastic (20/99, 20\%). Although these findings are similar to those reported in other studies reviewing muscle enlargement, ${ }^{2,3}$ the overall distribution of the causes does vary between published series. Trokel \& $\mathrm{Hilal}^{3}$ reported a higher incidence of vascular causes of NTR-EOME (12/24, 50\%) in their series, whereas Patrinely et $a l^{2}$ reported neoplastic causes $(28 / 60,46 \%)$ as the most commonly encountered diagnosis in their series.

The underlying diagnosis associated with NTR-EOME is usually made on the basis of orbital imaging, most commonly computed tomography (CT). Several studies have examined the CT findings most commonly
Department of

Ophthalmology, University Hospital of Coventry and Warwickshire, Coventry, UK

Correspondence:

F Shafi, Department of Ophthalmology, University Hospital of Coventry and Warwickshire, Clifford Bridge Road, Coventry, CV2 2DX, UK

Tel: 02476 965606; Fax: 02476966512. E-mail: farihashafi22@ gmail.com

Received: 23 May 2016 Accepted in revised form: 14 September 2016 Published online:

2 December 2016

Meeting presentation: Oral Presentation at British Oculoplastic Surgical Society Annual Meeting 2015 
found in NTR-EOME and have deduced some common patterns, which can help in formulating the underlying diagnosis. ${ }^{2,3}$ Although observations of the pattern of muscle involvement, muscle shape, and enhancement can lead to a limited differential diagnosis in some cases, no radiographic finding in itself seems to be pathognomonic. In almost all cases, correlative clinical findings are necessary to make a secure diagnosis. ${ }^{2,3}$

We report our outcomes of investigating and managing patients with NTR-EOME and explore the use of systemic imaging in further evaluating these cases. We feel the use of further systemic imaging might explain why our experience is different from that reported in literature, especially related to the higher incidence of malignancy and significantly lower incidence of inflammatory causes.

\section{Materials and methods}

A retrospective consecutive case series review was conducted of patients with NTR-EOME identified from orbital imaging and clinical features at a University Hospital (UHCW) between January 2007 and January 2015. Five hundred and ninety-three orbital CT scans were performed during this period, of which 116 (20\%) demonstrated EOME. The rectus muscles were designated as enlarged after comparison with other unaffected ipsilateral and contralateral muscles in the same view.

All patients underwent a comprehensive assessment, including a full clinical history and ocular examination. In particular, any history of previous malignancy was directly questioned at primary presentation. All patients had blood testing, including thyroid profile, which included free T3, free T4, and TSH levels but also TSHreceptor antibodies and thyroid peroxidase antibodies. All patients underwent baseline imaging of the orbits followed by further investigations, if required, depending on the findings on orbital imaging. Further systemic imaging was usually limited to the NTR-EOME group and sometimes helped in determining the most suitable diagnostic biopsy site. Our standard protocol for investigating suspected NTR-EOME is depicted in Figure 1.

Patient demographics, radiological features (including predominant EOM involved), and adjuvant tests including biopsy and final diagnosis were recorded for all patients. The cases were classified into the diagnostic groups of inflammatory, infective, primary tumor (local invasion or in situ origination, for example, lymphoma), metastatic tumor, vascular, or other. These diagnostic groups were independently analysed for trends and patterns.

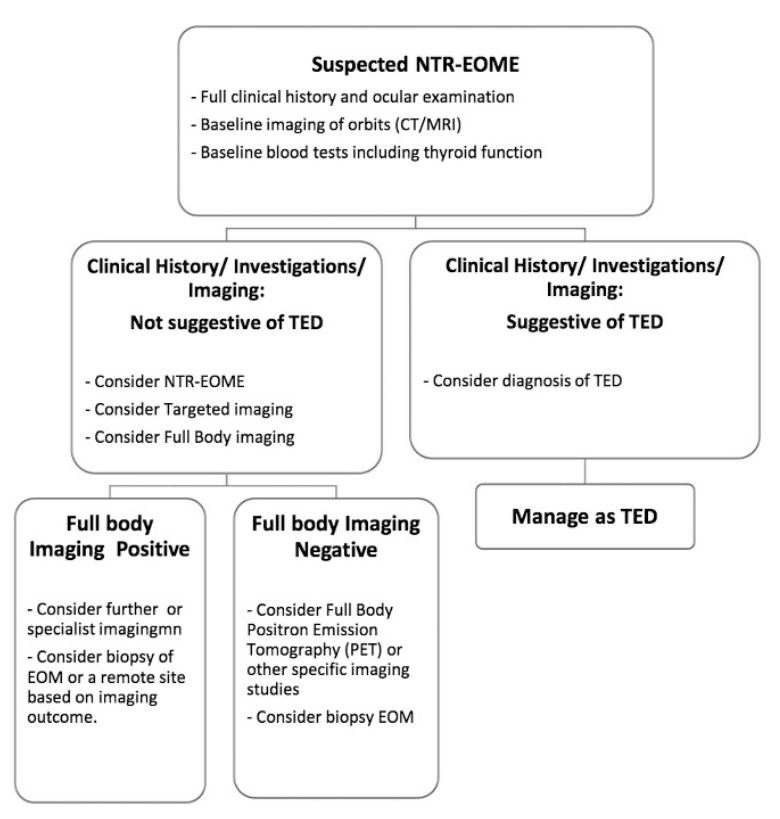

Figure 1 Management algorithm for NTR-EOME.

Ethics approval was not sought due to the retrospective nature of the study. Our research adheres to the tenets of the Declaration of Helsinki.

\section{Results}

\section{Patient demographics}

The mean age at presentation was 59.3 years (range 24-89 years). There was a slight female preponderance (10 female : 6 male).

\section{Diagnosis and extraocular muscle involvement}

The majority of cases in our series (14/16 cases, $87 \%)$ were associated with an underlying systemic neoplastic process. Table 1 outlines the diagnostic categories and the number of cases for each diagnostic category, comparing it with other published series of NTR-EOME. ${ }^{1-4}$ The most common primary or locally invasive tumour was lymphoma (5 cases). The majority of lymphoma cases involved either the superior rectus (SR) $(2 / 5)$ or the lateral rectus $(2 / 5)$, and only 1 of our 5 cases involved the inferior rectus. Adenocarcinoma from various sources was the most common metastatic tumour $(3 / 5)$, and the remaining 2 metastatic cases were associated with metastatic carcinoid tumour.

Our series also included four cases of EOME secondary to presumed paraneoplastic syndrome. Three of these cases were associated with breast carcinoma and one case was associated with thyroid papillary carcinoma. The diagnosis of presumed paraneoplastic syndrome causing 
Table 1 Diagnosis in 16 cases of NTR-EOME and comparison with other published NTR-EOME case series

\begin{tabular}{|c|c|c|c|c|c|c|}
\hline \multirow[t]{3}{*}{ Diagnosis } & \multicolumn{6}{|c|}{$\%$ of Cases } \\
\hline & \multicolumn{2}{|c|}{ Shafi et al $(\mathrm{n}=16)$} & \multirow{2}{*}{$\begin{array}{l}\text { Trokel \& Hilal } \\
\quad(\mathrm{n}=24)\end{array}$} & \multirow{2}{*}{$\begin{array}{l}\text { Rothfus \& Curtin } \\
\quad(\mathrm{n}=70)\end{array}$} & \multirow{2}{*}{$\begin{array}{l}\text { Patrinely et al } \\
\qquad(\mathrm{n}=60)\end{array}$} & \multirow{2}{*}{$\begin{array}{c}\text { Lacey et al } \\
\quad(\mathrm{n}=99)\end{array}$} \\
\hline & Number & $\%$ & & & & \\
\hline Neoplastic & 14 & 88 & 21 & 34 & 46 & 20 \\
\hline Primary/invasive lymphoma & 5 & & & & & \\
\hline Metastatic breast & 2 & & & & & \\
\hline Carcinoid & 2 & & & & & \\
\hline Ureteric TCC & 1 & & & & & \\
\hline Presumed paraneoplastic breast & 4 & & & & & \\
\hline Vascular & 1 & 6 & 50 & 10 & 13 & 24 \\
\hline Infective & 1 & 6 & & & 12 & 2 \\
\hline Inflammatory & 0 & 0 & 29 & 40 & 25 & 45 \\
\hline Other & 0 & 0 & & & 4 & 9 \\
\hline
\end{tabular}

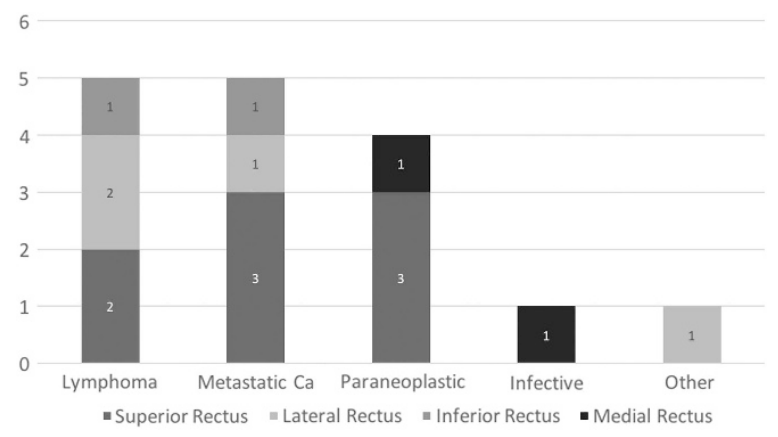

Figure 2 Extraocular muscle (EOM) involvement for each diagnosis.

NTR-EOME was made on the basis of evidence of resolution of EOME following treatment of the underlying carcinoma.

There was one infective case of medial rectus enlargement associated with orbital cellulitis, which resolved promptly with systemic antibiotics. The final case of lateral rectus enlargement was secondary to a dural-venous shunt causing hydrocephalus. Extraocular muscle resolved with treatment of the hydrocephalus after performing an endoscopic third ventricle ventriculostomy.

All cases involved predominant enlargement of a single rectus muscle. The most commonly involved EOM was the SR $(8 / 16$ cases, $50 \%)$ followed by the lateral rectus $(4 / 16,25 \%)$. All cases of SR enlargement were associated with an underlying neoplastic cause. (Figure 2)

\section{Imaging}

All patients underwent baseline imaging of the orbits followed by systemic imaging, if indicated based on the clinical index of suspicion of possible underlying systemic pathology. All patients exhibited diffuse muscle belly enlargement with no obvious discerning features apparent between the different underlying diagnoses (Table 2). Of the 16 patients, 14 underwent further systemic imaging. The majority (13/14) underwent full body CT (FBCT), which included imaging of the head, chest, abdomen, and pelvis. One patient presenting with a coincidental breast mass underwent mammography instead, which confirmed a breast mass. Positive relevant systemic radiological findings were detected in 12/14 cases $(11 / 13$ positive on FBCT, $1 / 1$ positive on mammography). Of the two patients with negative findings on FBCT, one underwent further full body positron emission tomography-computed tomography (FBPET-CT), which detected a thyroid nodule. Biopsy of this nodule confirmed thyroid papillary carcinoma. The second patient with negative FBCT had undergone systemic imaging as a staging scan following an orbital biopsy positive for lymphoma. Of the 11 patients with positive findings on FBCT, 3 underwent further full body PET-CT (2 for staging of disease and 1 for monitoring for recurrence; Figure 3a).

\section{Biopsy}

In total, 14/16 underwent biopsy from an orbital and/or systemic site. Of the 16 patients, 4 underwent orbital biopsy, 8 patients underwent biopsy from a systemic site, and 3 patients underwent orbital biopsy followed by biopsy elsewhere. Two patients had no biopsy or FBCT as this was not clinically indicated in these cases (NTREOME caused by orbital cellulitis and hydrocephalus respectively; Figure 3b). 


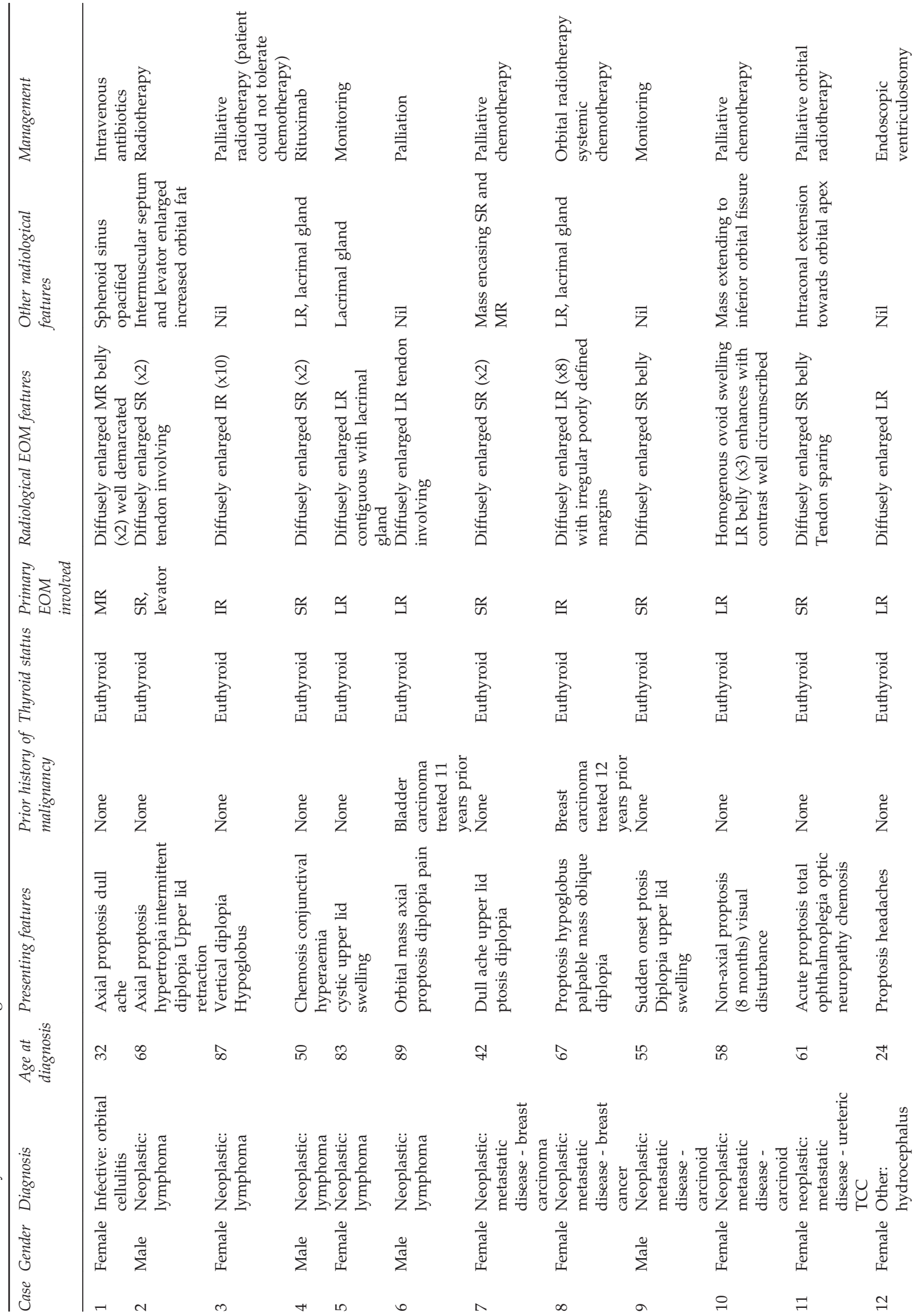




\section{Follow-up}

Mean follow-up was 3.2 years (range 3 months to 5.5 years). Four patients (25\%) died within 3 years of followup from time of presentation due to disseminated systemic malignancy. The underlying diagnoses in these patients were lymphoma (2 patients), metastatic carcinoid tumour (1 patient), and advanced metastatic ureteric transitional cell carcinoma (1 patient; Figure $3 b$ ).

\section{Further management}

Subsequent management of each patient was dependent on the underlying diagnosis. This is summarised in Table 2.

\section{Conclusion}

Clinicians are often faced with the diagnostic dilemma of the orbital patient with an isolated, enlarged extraocular muscle on CT scan, normal thyroid function tests, and no clinical findings, suggestive of thyroid eye disease. These cases can be classified as NTR-EOME. Studies reviewing diagnoses in NTR-EOME have reported contradictory patterns in certain disease groups, which has further contributed to the complexity of diagnosing these cases.

Neoplastic involvement of the extraocular muscles may be caused by local infiltration by adjacent tumours, including primary orbital tumours and secondary neoplasms from periorbital sites, or by metastases from distant sites. Tumours primarily involving extraocular muscles are extremely rare. These can include rhabdomyosarcoma, lymphoma, meningiomas, sinus tumours, granular cell tumour, and alveolar soft part sarcoma. ${ }^{3-6}$ In our study, neoplastic causes accounted for the majority of NTR-EOME cases (14/16, 87\%). Of these, five cases were caused by orbital lymphoma, a further five cases had discrete metastases to an extraocular muscle from a systemic carcinoma, and four cases had presumed paraneoplastic syndrome secondary to a systemic carcinoma at a remote site.

Patrinely et $a l^{2}$ reported primary tumours as comprising the largest diagnostic category in their series of $(16 / 60)$ cases. However, they do speculate that myositic pseudotumour was actually the more common diagnosis and that their tertiary referral pattern may have selected for a higher percentage of unusual oncology cases. In their series, they found several strong patterns within this group. Cases were frequently unilateral and involved single muscles. Lymphoma involved the SR and lateral rectus group, which is in agreement with Hornblass et $a l^{\prime} \mathrm{s}^{6}$ report of six of seven muscle lymphoma cases originating in the SR group. We have also found that extraocular muscle lymphoma predominantly involves 
a

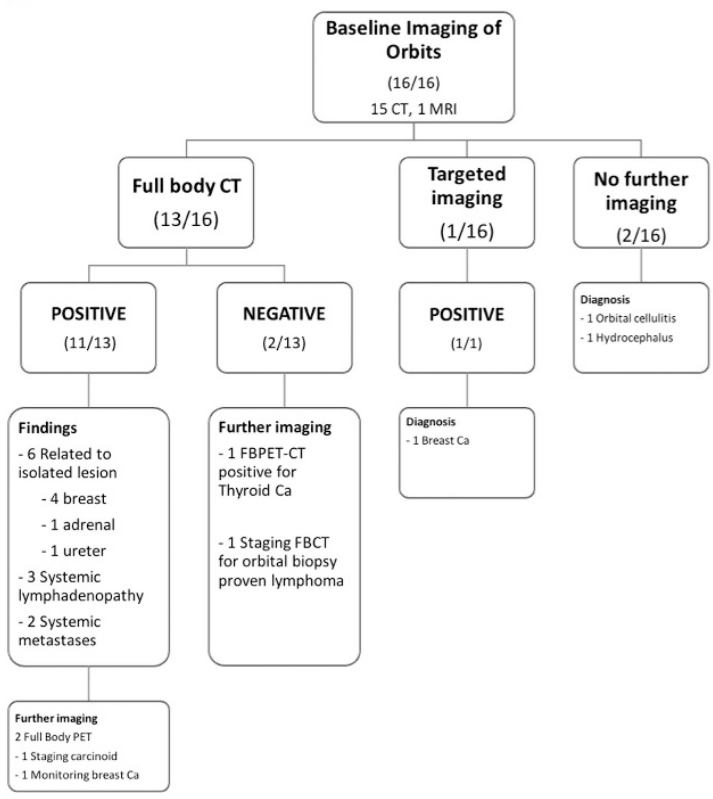

b

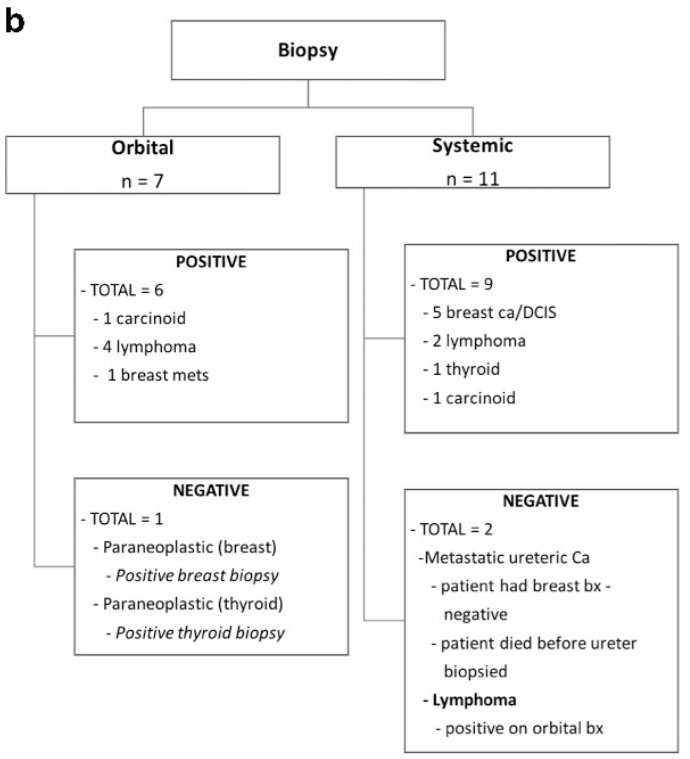

Figure 3 Patient Investigations. (a) Imaging flow chart. (b) Biopsy site and findings.

the SR (2/5) and lateral rectus (2/5). Only 1 of our cases involved the inferior rectus. In contrast to our findings, Lacey et $a l^{1}$ reported neoplastic processes as a cause of EOM enlargement in only $18 \%$ of their cases, of which, 8 had invasion from adjacent orbital neoplasms and 12 cases had metastases to extraocular muscles.

In our series, the primary tumour site for metastasis to the EOM was breast $(2 / 5)$, carcinoid tumour $(2 / 5)$, and ureteric transitional cell carcinoma (1/5). Patrinely et $a l^{2}$ also report breast adenocarcinoma as being most frequent site of primary tumour for extraocular muscle metastasis. A review of the literature, however, reports that breast and cutaneous melanoma are the most common primary origins for discrete muscle metastasis. ${ }^{1,7}$ In their series,

Lacey et $a l^{1}$ reported 7 cutaneous melanomas and 3 breast carcinomas. Skin and breast were also the most common primary sites for metastasis to muscles, reported in the review by Capone and Slamovits. ${ }^{7}$ These trends were not noted in our own study. We encountered no cases of metastasis from skin/cutaneous melanomas. The frequency of rectus involvement in Capone and Slamovits study was medial $(39 \%)$, lateral $(33 \%)$, superior $(16 \%)$, and inferior $(12 \%)$. The distribution of rectus involvement in our series was SR $(3 / 5,60 \%)$, lateral rectus $(1 / 5,20 \%)$, and inferior rectus $(1 / 5,20 \%)$.

The majority of patients with metastases to extraocular muscle $(58 \%)$ have been reported to have a diagnosis of primary malignancy at presentation. ${ }^{8}$ This is contrary to our findings, where the diagnosis of the primary tumour at presentation was only known in one case of carcinoid tumour $(1 / 5,20 \%)$. One of the patients with metastatic breast disease had previously been treated for breast carcinoma, but had been discharged from medical care following successful treatment of the tumour. It is more than likely that this patient presented with recurrence of her original breast carcinoma with subsequent extraocular muscle metastasis. Metastases to extraocular muscle occurs late in the course of a systemic tumour and therefore the prognosis at presentation with orbital involvement is poor. ${ }^{9-11}$ This is in keeping with our findings as two patients with metastatic EOME in our series died within 3 years of presentation-one patient from metastatic carcinoid tumour and another patient from advanced ureteric transitional call carcinoma.

Vascular etiologies of rectus muscle enlargement include arteriovenous malformations, carotid-cavernous sinus fistula, dural-venous shunts, and angiomas. These conditions are known to cause unilateral, moderate, and uniform enlargement of the horizontal rectus muscles, but single muscle involvement can also occur. The muscle expands because of increased venous pressure and vascular distention. ${ }^{12}$ In our series, we encountered 1 case of a dural-venous shunt causing hydrocephalus and resultant lateral rectus enlargement. The EOME resolved with emergency endoscopic third ventricle ventriculostomy.

Although extraocular muscles may be affected as part of a generalized orbital infection by any microorganisms, contiguous involvement from bacterial sinusitis is by far the most common infection affecting extraocular 
muscles. ${ }^{13}$ The medial rectus is more frequently involved in infectious cases due to its close proximity to the ethmoid sinus. We had one case of orbital cellulitis with associated medial rectus, which resolved promptly with systemic antibiotic treatment.

Paraneoplastic syndromes are rare disorders that are triggered by an altered immune system response to a neoplasm. They are defined as clinical syndromes involving non-metastatic systemic effects that accompany malignant disease. Recently, there have been reports describing paraneoplastic syndromes causing EOME, but this process is still neither well understood nor well defined. ${ }^{14,18}$ Swash in 1974 reported external ophthalmoplegia in a patient with bladder carcinoma. ${ }^{14}$ The patient developed a progressive acute necrotizing myopathy affecting the skeletal and extraocular muscles. On biopsy, the extraocular muscles showed marked variation in shape and size, and there was evidence of endomysial edema and hyaline degeneration of muscle fibers. ${ }^{14}$ Harris et al ${ }^{15}$ reported an orbital myositis in association with a paraneoplastic syndrome in a patient with non-Hodgkin's lymphoma. A similar case of bilateral extraocular muscle inflammatory involvement has been described in a patient with metastatic paraganglioma, ${ }^{16}$ and in four cases of retinoblastoma associated with muscle damage, but no cancer cells invading the muscle. ${ }^{17}$ We have previously published one of the cases in our series, ${ }^{18}$ A 65-year-old male with SR mass was found to have a breast mass on systemic imaging and biopsy confirmed breast adenocarcinoma. The patient underwent a right radical mastectomy following which his proptosis resolved and repeat imaging shows reduction in the size of the SR muscle.

In paraneoplastic syndrome, it is thought that an antibody directed against the tumour cross-reacts with antigens on extraocular muscles, leading to inflammation and tissue destruction. We have now had experience of managing three further cases of presumed paraneoplastic EOME, two secondary to breast carcinoma, and one secondary to thyroid papillary carcinoma. One patient with MR enlargement secondary to breast carcinoma was originally diagnosed as myositis following a medial rectus muscle biopsy at another institution. The myositis remained recalcitrant and the patient required low-dose steroid treatment due to break through inflammation on stopping treatment. Almost 10 years later, the patient was diagnosed with bilateral breast carcinoma and following wide local excision the medial rectus enlargement was resolved. The second patient with SR enlargement secondary to breast carcinoma was diagnosed with a breast lesion following FBCT, and in this case, the EOME also resolved following excision of the underlying tumour. The FBPET-CT did not show any activity of the $\mathrm{SR}$ and this feature can distinguish paraneoplastic EOME from metastatic EOME. In the patient with thyroid papillary carcinoma, the SR mass preceded the radiological detection of the thyroid mass by 4 years. Initial biopsy had shown non-specific inflammation and FBCT was also negative for any systemic lesion. The patient presented 4 years later with an enlarged SR on the contralateral side. FBPET-CT showed no increased activity in the enlarged SR but did show increased activity in an enlarged thyroid gland, and subsequent biopsy confirmed papillary carcinoma. Paraneoplastic syndromes can precede radiologically detectable masses, ${ }^{19}$ hence negative systemic imaging cannot reliably exclude malignancy and such patients should be monitored at regular intervals, and repeat imaging should be considered if no other cause for EOME can be determined.

Several studies report myositis as the most common cause of NTR-EOME. In the review by Lacey et al ${ }^{1}$ acute myositis was the cause of $43 \%$ of all cases of NTR-EOME. Interestingly, we describe no cases of myositis in our series. This may be partly accounted for by the cases of presumed paraneoplastic syndrome, which would likely have otherwise been initially diagnosed as myositis if systemic imaging been performed.

Our series has clearly shown that the isolated EOME can be a presenting feature of underlying systemic malignancy, and an appropriate systemic workup should be considered. In the majority of these cases, a FBCT scan can help in identifying or ruling out a primary systemic cause. Full body PET-CT is best reserved for cases negative on whole-body CT scan, where the clinical index of suspicion for a systemic aetiology is strong, or for staging and monitoring disease. Imaging can also be targeted to specific areas based on the clinical index of suspicion, for example, breast mammography, if there is a breast mass, octreotide scanning in carcinoid tumours. One must be prepared to revisit the initial diagnosis and investigate further in case of recurrences or failure to respond to treatment. This includes cases of recalcitrant myositis, which keeps relapsing on withdrawal of immunosuppression. It should be highlighted that the systemic imaging should only be used judiciously as there is an associated radiation risk for the patient as well as added expense associated with the investigation.

In summary, we have reviewed and documented a spectrum of cases of NTR-EOME, of which the majority were associated with systemic neoplasia. The higher incidence of neoplasia in our cohort could be attributable to our protocol for investigating NTR-EOME. This would also explain our apparent lack of cases with idiopathic orbital myositis, which is often a diagnosis of exclusion. The proportion of NTR-EOME associated with underlying malignancy may have been underestimated in previous studies, or our population base might be 
significantly different to previous studies. In our experience, all cases should be viewed with a high index of clinical suspicion for systemic neoplasia, especially when the SR is involved. An appropriate systemic workup should be performed to exclude a potential occult primary when all other preliminary tests including orbital biopsy are negative. Systemic imaging should be considered in all cases of isolated NTR-EOME as this diagnosis can be associated with significant mortality (25\%).

\section{Summary}

What was known before

- Extraocular muscle enlargement (EOME) is most commonly associated with thyroid eye disease. The EOME can also be the resultant manifestation of several other non-thyroid-related conditions, including inflammatory, vascular, neoplastic, infectious, neuromuscular, and metabolic disorders (NTR-EOME).

\section{What this study adds}

- All cases of NTR-EOME should be viewed with a high level of clinical suspicion for systemic neoplasia, especially when the SR is involved. Non-thyroid-related EOME can be associated with significant mortality ( $25 \%)$, hence warrants prompt and thorough systemic investigation.

\section{Conflict of interest}

The authors declare no conflict of interest.

\section{References}

1 Lacey B, Chang W, Rootman J. Nonthyroid causes of extraocular muscle disease. Surv Ophthalmol 1999; 44: 187-213.

2 Patrinely J, Osborn A, Anderson R, Whiting AS. Computed tomographic features of nonthyroid extraocular muscle enlargement. Ophthalmology 1989; 96: 1038-1047.

3 Trokel S, Hilal S. Recognition and differential diagnosis of enlarged extraocular muscles in computed tomography. Am J Ophthalmol 1979; 87: 503-512.
4 Rothfus WE, Curtin HD. Extraocular muscle enlargement: a CT review. Radiology 1984; 151: 677-681.

5 Peyster RG, Hoover ED. Computerized Tomography in Orbital Disease and Neuro-ophthalmology. Year Book Medical Publishers: Chicago, IL, USA, 1984; 23-116.

6 Hornblass A, Jakobiec FA, Reifler DM, Mines J. Orbital lymphoid tumors located predominantly within extraocular muscles. Ophthalmology 1987; 94: 688-697.

7 Capone Jr A, Slamovits TL. Discrete metastasis of solid tumors to extraocular muscles. Arch Ophthalmol 1990; 108: 237-243.

8 Goldberg RA, Rootman J, Cline RA. Tumors metastatic to the orbit: a changing picture. Surv Ophthalmol 1990; 35: 1-24.

9 Volpe NJ, Albert DM. Metastatic and secondary orbital tumors. In: Albert DM, Jakobiec FA (eds). Principles and Practice of Ophthalmology Vol 3. WB Saunders: Philadelphia, PA, USA, 1994; 2027-2050.

10 Harstein ME, Grove Jr AS, Woog JJ. The role of the integrin family of adhesion molecules in the development of tumors metastatic to the orbit. Ophthalmic Plast Reconstr Surg 1997; 13: 227-238.

11 Henderson JW. Metastatic carcinomas. In: Henderson JW (ed). Orbital Tumors. Raven Press: New York, NY, USA, 1994; 361-375.

12 Grove AS Jr. The dural shunt syndrome. pathophysiology and clinical course. Ophthalmology 1983; 91: 31-44.

13 Towbin R, Han BK, Kaufman RA, Burke M. Postseptal cellulitis: CT in diagnosis and management. Radiology 1986; 158: 735-737.

14 Swash M. Acute fatal carcinomatous neuromyopathy. Arch Neurol 1974; 30: 324-326.

15 Harris G. Orbital myositis as a paraneoplastic syndrome. Arch Ophthalmol 1994; 112: 380.

16 Spraul CW, Lang GE, Lange GK. Orbital myopathy in metastatic malignant paraganglioma: a paraneoplastic syndrome? Klin Monatsbl Augenheilkd 1996; 209: 153-157.

17 Finol HJ, Marquez A, Navas E, de Navas NR. Extraocular muscle ultrastructural pathology in the paraneoplastic phenomenon associated with retinoblastoma J Exp Clin Cancer Res 2001; 20: 281-285.

18 Mehta P, Chickadasarahally S, Hedley N, Ahluwalia H. Extraocular muscle enlargement as a paraneoplastic effect of breast carcinoma in a male patient. Ophthal Plast Reconstr Surg 2011; 27: e146-e147.

19 Hart I. Phenotypic variants of autoimmune peripheral nerve hyperexcitability Brain 2002; 125: 1887-1895. 